\title{
Visibilidad mediática-social de las revistas académicas iberoamericanas de artes y humanidades en Emerging Source Citation Index: una aproximación altmétrica
}

\author{
Juan José Prieto-Gutiérrez \\ Universidad Complutense de Madrid, Madrid, España \\ jujpriet@ucm.es \\ Joel Alhuay-Quispe \\ Universidad Nacional Mayor de San Marcos, Vicerrectorado de Investigación y Postgrado, Dirección de \\ Evaluación e Impacto, Lima, Perú \\ joel.alhuay@unmsm.edu.pe
}

\begin{abstract}
DOI: https://doi.org/10.26512/rici.v13.n3.32734
\end{abstract}
Recebido/Recibido/Received: 2020-06-10

Aceitado/Aceptado/Accepted: 2020-08-05

Resumen: El nuevo producto de Web of Science, la base de datos Emerging Sources Citation Index (ESCI), crece anualmente de una forma notable. En la actualidad cuenta con más de 8,000 revistas de todas las disciplinas académicas. El artículo busca analizar la visibilidad de las revistas Iberoamericanas de las áreas de Artes y Humanidades $(A \& H)$ calificadas en este índice a partir de caracterización de las subcategorías, editoriales, idiomas de publicación y la actividad en medios sociales de las revistas evaluadas. Se emplea métodos de análisis descriptivo basado en datos de la plataforma Altmetric.com para evaluar la repercusión mediática de 442 revistas de América Latina, El Caribe, España y Portugal. Se concluye que más de la mitad de las subcategorías (17 de 26) de A\&H incluyen revistas de procedencia iberoamericana. Destacando 2 de ellas al presentar una importante cobertura global superior al 55\%: Literature, Romance (71,79\%) y Classics (56,25\%) y en 3 de ellas superior al 40\%: Architecture (43,75\%); History (46,72\%) y Medieval \& Renaissance Studies (45,94\%). La presencia del inglés como un posible idioma de publicación en más de la mitad de las revistas analizadas indica el grado de visibilidad de las mismas. La mayoría de las revistas analizadas tienen repercusión en redes sociales, siendo Facebook y Twitter las más activas, en donde las interacciones y comentarios, por parte de países externos a Iberoamérica, relacionado con el contenido de las revistas estudiadas contribuyen a afirmar la aceptable visibilidad de las revistas de A\&H Iberoamericanas.

Palabras clave: Emerging Sources Citation Index. Web of Science. Revista científica. Visibilidad mediática. Almetria.

Visibilidade social dos periódicos científicos de Iberoamérica em Artes e Humanidades do ESCI-WoS (Emerging Source Citation Index): uma abordagem altmétrica

Resumo: O banco de dados Emerging Sources Citation Index (ESCl) é novo produto do Web of Science, com crescimento notável a cada ano. Atualmente possui mais de 8,000 periódicos de todas as disciplinas acadêmicas. $O$ objetivo deste trabalho é analisar a visibilidade das revistas ibero-americanas na área de Arte e Humanidades $(\mathrm{A} \& H)$ desse índice partindo da caracterização das subcategorias temáticas, editoriais, linguagens de publicação e atividade altmétrica em mídias sociais. Métodos de análise descritivos baseados em dados da Altmetric.com são utilizados para avaliar o impacto de 442 periódicos de América Latina, Caribe, Espanha e Portugal na mídias sociais. Conclui-se que mais da metade das subcategorias de A\&H (17 de 26) inclui periódicos de origem ibero-americana. 2 deles se destacam, com cobertura global significativa de mais de 55\%: Literatura, Romance (71,79\%) e Clássicos (56,25\%) e em 3 de eles estão acima de 40\%: arquitetura (43,75\%); História (46,72\%) e Estudos Medievais e Renascentistas (45,94\%). A presença do inglês como possível idioma de publicação em mais da metade das revistas analisadas indica o grau de visibilidade delas. 
Um grande número de revistas analisadas é encontrado nas redes sociais, sendo o Facebook e o Twitter os mais ativos, onde interações e comentários, por países fora da Ibero-América, relacionados ao conteúdo das revistas estudadas contribuem para afirmar a aceitável visibilidade das revistas da A\&H Ibero-americanas.

Palavras-chave: Emerging Sources Citation Index. Web of Science. Periódico científico. Visibilidade nas redes sociais. Altmetria.

Social visibility by Ibero-American scholarly journals in Arts and Humanities from ESCI-WoS (Emerging Source Citation Index): an altmetric approach

Abstract: Emerging Sources Citation Index (ESCl) as new Web of Science product database, grows remarkably every year. It currently has more than 8,000 journals across different academic disciplines. This work aims to analyze visibility from Iberoamerican journals in Art and Humanities (A\&H) areas based on characterization of research sub-categories, publishers, publication languages and impact activity in social media. Descriptive analysis methods were carried out on data from Altmetric.com platform to social mediatic impact assessment of 442 scholarly journals from Latin America, the Caribbean, Spain and Portugal. It is concluded that more than half of A\&H sub-categories (17 of 26) include journals of Ibero-American origin. 2 of them standing out, with significant global coverage of over 55\%: Literature, Romance $(71.79 \%)$ and Classics (56.25\%) and 3 of the over 40\%: Architecture (43.75\%); History (46.72\%) and Medieval \& Renaissance Studies (45.94\%). The presence of English as a possible publication language in more than half of the analyzed journals indicates the degree of visibility of the same. A large number of the journals analyzed are found on social networks, with Facebook and Twitter being the most active, where interactions and comments by countries outside IberoAmerica related to the content of the journals studied contribute to affirm the acceptable visibility of A\&H Ibero-Americana journals.

Keywords: Emerging Sources Citation Index. Scholarly journals. Visibility on social media. Altmetrics.

\section{Introducción}

El propósito final de una investigación es la publicación y comunicación de la misma, pero esta actividad varía enormemente en función de la disciplina científica en la que se trabaja; en consecuencia, no es lo mismo publicar y comunicar trabajos relacionados con las ciencias exactas y naturales que con las artes y humanidades (GARFIELF, 1980). Mientras que los académicos de las artes y humanidades suelen crear contenidos en formatos sui generis (como podrían ser las piezas de arte o los poemas) que difícilmente pueden ser cubiertos por las bases de datos de literatura académica. Por ejemplo, un historiador del arte o un arquitecto no pueden citar formalmente edificios de Frank Gehry o las obras de Richard Serra; en cambio, al hablar del Coronavirus es factible emplear decenas de referencias bibliográficas provenientes de revistas científicas, repositorios de pre-prints, bases de datos especializadas y globales, etc.

En la misma línea, aquellos pertenecientes a las áreas de ciencias, tecnologías, ingenierías y matemáticas, conocidas como STEM (del inglés Science, Technology, Engineering \& Mathematics) buscan una rápida comunicación sobre los avances y hallazgos y, para ello, los autores redactan artículos para publicar en las revistas especializadas del sector. En cambio, los canales de comunicación y las prácticas de publicación de las artes y humanidades suelen ser más variadas; además de las revistas académicas se les pueden sumar otros medios de publicación como libros, capítulos de libros, entre otros (NEDERHOF, 2006), y más recientemente mediante las redes sociales (THELWALL, 2018), que muy a menudo se focalizan en un área geográfica determinada por el 
empleo de la lengua local (ARCHAMBAULT et al, 2006) y por tanto, el interés a la colaboración internacional es reducido, limitando la visibilidad de los resultados de investigación humanística y artística.

Aunque los artículos en coautoría han aumentado en numerosas disciplinas, los patrones de autoría varían enormemente de unas áreas a otras (JABBEHDARI; WALSH, 2017). Los humanistas y académicos de las artes prefieren publicar en solitario (BUCHWALD, 1999) limitando esa visibilidad, mientras que los científicos tienden a firmar sus investigaciones mediante grupos (BRODERICK; CASADEVALL, 2019). Por tanto, el concepto de visibilidad puede entenderse, en la aplicación práctica, desde dos perspectivas: a) la presencia de las revistas en índices de evaluación internacionales y b) la presencia en Internet de las revistas y sus contenidos electrónicos.

En relación con la primera alternativa, en las últimas décadas, los estudios bibliométricos de las ciencias y las ciencias sociales han ganado más legitimidad que esfuerzos similares para explorar las artes y humanidades. (LEYDESDORFF; SALAH, 2010). Como es sabido, uno de los indicadores de visibilidad que cuantifica a las revistas científicas es el factor de impacto que, en los inicios ofrecía la institución ISI (Institute for Scientific Information) de Thomson Reuters, y luego por Clarivate Analytics, a través varias bases de datos disciplinares integradas en la plataforma Web of Science: Science Citation Index Expanded (SCl-Expanded) desde 1945; Social Sciences Citation Index (SSCI) desde 1956; Arts and Humanities Citation Index (AHCI) desde 1975 y Emerging Sources Citation Index (ESCl) desde 2015. Con estas dos colecciones ( $\mathrm{SCl}$ y SSCl) se conforman los rankings de revistas globales que se reporta anualmente a través de los JCR (Journal Citation Reports).

En este punto, igualmente aparece una desigual cobertura en cuanto a las áreas científicas y la procedencia geográfica de la publicación. Por lo que, el uso de las citas en las Artes y Humanidades puede considerarse un elemento desfavorable para calcular el factor de impacto comparado con las revistas inmersas en las áreas STEM. La tradición de citas en las humanidades no es tan fuerte como en las ciencias y por tanto la comunicación académica difiere; tanto es así, que en los años ochenta del siglo pasado se afirmaba que la calidad de las publicaciones no debía solamente abordarse mediante métodos cuantitativos basados en las citas bibliográficas (FINKENSTAEDT, 1980). Pero en la actualidad, el índice de impacto que se genera en la totalidad de las revistas indexadas en cualquiera de las bases de datos sigue siendo el indicador más destacado de calidad y, por ende, de visibilidad de, artículo, revista y autor.

Probablemente debido a estas disparidades, durante los últimos años han crecido los estudios e investigaciones enfocados sobre las revistas del área de Artes y Humanidades (LEYDESDORFF; HAMMARFELT; SALAH, 2011) (WANG; HO, 2017) (HAMMARFELT, 2016); más específicamente aquellos enfocados sobre una región geográfica o país (NWAGWU; EGBON, 2011) (HEILAND; HUBER; KANTER, 2014). 


\section{2. $\mathrm{ESCl}$ y los nuevos productos de la colección de Web of Science}

Indudablemente, todos los productos provenientes de la Web of Science (WoS) implican sinónimo de calidad y por ello, publicar en las bases de datos de su colección ostenta valoraciones favorables por la comunidad científica. Esta situación ha generado que en los últimos años han aparecido nuevos productos que diversifican los contenidos de las colecciones tradicionales del sistema de citación global de revistas más antiguo y afamado. Entre las recientes colecciones aparecidas se incluye la Chinese Science Citation Database, en 2008, como la primera colección de habla no inglesa; años más tarde, en 2013 se incluye a SciELO Citation Index, una colección de las revistas calificadas en la red SciELO; en 2014 se integra otra colección asiática, la Korean Citation Index $(\mathrm{KCl})$ y en noviembre de 2015 comunicó la liberación de una nueva colección como parte del "core": Emerging Source Citation Index (ESCI). Esta colección de WoS inicia en 2015 con 2.400 revistas aproximadamente y a finales del año 2019 posee cerca de 8.000, sin presentar distinción por materias de Ciencias, Ciencias Sociales, Arte y Humanidades, etc. como presentan las otras bases de datos más específicas de Clarivate Analytics. No cabe duda que estas apariciones obedecen a la presencia de nuevos competidores cada vez más orientados a la cobertura de contenidos de acceso abierto y regionales como Dimensions, Lens, Microsoft Academic Search, Semantic Scholar, Scite, Wizdom, etc.

WoS como base de datos de larga trayectoria ofrece amplia expectativa de las revistas a nivel global para que los autores e instituciones puedan monitorear y comparar el incremento de su prestigio al estar visibles desde la plataforma científica más empleada por los investigadores. En la misma línea, la calificación en $\mathrm{ESCl}$ ha comenzado a tenerse en cuenta por algunas agencias de evaluación de diversos países debido a la calidad y a las altas posibilidades que poseen las revistas que allí transitan de dar el "salto" a categorías superiores como podrían ser $\mathrm{SCl}, \mathrm{SSCl}$ o $\mathrm{AHCl}$. Para ello necesitan mantener la más alta calidad de publicaciones académicas para ser indexadas internacionalmente y cumplir con las expectativas de sus autores potenciales (BESHYAH, 2019).

Pocos estudios se han realizado sobre las revistas incluidas en la base de datos emergente, destacando algunos sobre ciertas disciplinas científicas, como las revistas Iberoamericanas de Educación (REPISO; JIMÉNEZ-CONTRERAS; AGUADED, 2017); revistas de Comunicación (MIQUELSEGARRA, 2018); revistas de Educación y Comunicación (COMUNICAR, 2018) y revistas del área de Bibliotecología y Ciencia de la Información (REPISO, 2019) o sobre áreas geográficas como las revistas $\mathrm{ESCl}$ de procedencia rusa (Москалева у Писляков, 2017) о españolas (Ruiz Pérez у Jiménez-Contreras, 2019).

Como se observa, la literatura consultada reporta todavía poca información de investigaciones basado en $\mathrm{ESCl}$ con atención a las diversas áreas de conocimiento o las grandes materias de 
investigación. Por tanto, al no haberse realizado análisis de grandes materias como las Artes y Humanidades, existe un gran espacio para seguir investigando y vacío de conocimiento al que se pretende aportar con este estudio.

\section{Repercusión altmétrica en revistas}

La segunda alternativa, indicada en el acápite introductorio, en relación con el aumento de la visibilidad de las revistas y su producción científica se centra en el papel de las redes sociales como nuevos vehículos para la difusión y el impacto de la ciencia en la sociedad. Dicha visibilidad y presencia de una revista en las redes sociales puede medirse en base a las estadísticas de uso de los contenidos (vistas de páginas, descargas de ficheros) que influyen en la repercusión de los artículos en plataformas de redes sociales (compartidos, publicaciones) y que posteriormente, se concretan en impacto científico medido por las citas recibidas (VILCHEZ-ROMAN; HUAMANDELGADO; ALHUAY-QUISPE, 2020).

Los estudios centrados en determinar los mecanismos y comportamientos de la difusión de los resultados de investigación se conocen como estudios de repercusión social-mediática o de actividad altmétrica (abreviatura de métricas alternativas). Estos fueron introducidos de una forma estable en el año 2010 (PRIEM et. al., 2010) como una forma alternativa de medir los impactos más amplios de la investigación en las redes sociales a través de diferentes herramientas (PRIEM; PIWOWAR; HEMMINGER, 2012) que día a día van siendo más usadas por la sociedad y con actitud positiva por la comunidad cientifica y academica (SEGADO-BOJ; MARTíN-QUEVEDO; PRIETOGUTIÉRREZ, 2018). Siendo posible rastrear el impacto 'en tiempo real' de los resultados científicos mediante la exploración de los recursos compartidos, discusiones, reseñas, los tweets, menciones de publicaciones científicas y fuentes en las redes sociales, marcadores y otras acciones como los "likes", comentarios, guardar, etc. (WOUTERS; COSTAS, 2012).

Actualmente, la herramienta más empleada y que más credibilidad ofrece es la plataforma Altmetric.com. Esta, monitorea varias redes sociales simultáneamente, destacando: YouTube, Google, Reddit, Twitter, Facebook, Mendeley, ResearchGate, Reddit, LinkedIn, blogs, noticias, etc. Las menciones, visitas, descargas, comentarios y difusiones, entre otras acciones, generan un indicador numérico que aumenta cada vez que el trabajo aparece en algunos de los medios indicados, despertando un cierto interés y aceptación. El uso de estas herramientas abre una ventana valiosa en los procesos académicos: evaluación de autores y revisión de las revistas, sus artículos y las propias disciplinas científicas (SUGIMOTO et al., 2017).

En relación con las disciplinas académicas, son las Artes y Humanidades las que probablemente pueden generar un impacto social amplio y dinamizar debates públicos, no siempre tenido en cuenta. La visibilidad de las revistas de este sector no se debe limitar al estudio de los índices de 
citas, como en otras áreas. Las prácticas de los investigadores pueden ser poco convencionales, por ejemplo, citando capítulos de libros y un amplio espectro de libros de literatura, traduciendo sus obras a varios idiomas, etc. Por tanto, dada la falta de información actual sobre sobre el comportamiento de las revistas y autores del área de Arte y Humanidades, el artículo actual investiga la visibilidad de las revistas científicas incluidas en el índice $\mathrm{ESCl}$ en Iberoamérica complementando la información empleada con datos proporcionados por el principal recolector altmetric actual, Altmetric.com.

Medularmente, los objetivos que se persiguen en esta investigación son dos: a) caracterizar las revistas Iberoamericanas en ESCl de Artes y Humanidades (cobertura, idioma, tipología editorial) y b) evaluar la repercusión mediática (actividad altmétrica) de tales revistas.

\section{Metodología}

\subsection{Diseño y enfoque}

Se trata de un estudio descriptivo, observacional y correlacional de corte temporal transversal, que emplea el enfoque de análisis métrico basado en indicadores altmétricos a partir de una muestra de tipo censal que incluye a revistas iberoamericanas de Artes y Humanidades indizadas en ESCI-WoS.

\subsection{Unidades de análisis}

El artículo analiza publicaciones periódicas académicas editadas y publicadas por instituciones de países de América Latina, El Caribe, Brasil, España y Portugal, que se encuentran indizadas en la colección ESCI de Web of Science.

Con fecha de septiembre del 2019, se identificaron 7,852 revistas indizadas en la denominada "colección emergente" de WoS, de las cuales 1,387 revistas corresponden a las áreas de Artes y Humanidades, y de estas, 442 son de procedencia de países iberoamericanos, siendo esta cantidad nuestra muestra final para evaluación.

\subsection{Colecta de datos}

Las unidades de análisis fueron extraídas a partir de una base de datos de las revistas ESCI proporcionada por Clarivate Analytics para fines de investigación y fue complementada con información recuperada del sitio Master Journal List (MJL) - Web of Science.

Los datos correspondientes a los análisis complementarios de impacto mediático o datos altmétricos fueron obtenidos a partir del recurso "Explorer" de acceso para investigadores de Altmetric.com., agrupados en cinco fuentes: Social media (Twitter, Facebook, Google+, Reddit Posts, Sina Weibo, Pinterest, Linkedin), Policy and patents (patents, policy documents), News and 
blogs (news, blogs), Academic sources (research highlights, peer reviews) y otros recursos (Wikipedia, Videos, Q\&A posts).

Para la investigación se utilizan los datos de menciones en social media, específicamente en Twitter y Facebook; por considerarse los más representativos y dada la escasa o nula presencia en las otras fuentes de redes sociales como Google+ o Linkedln. Otros datos considerados en la recolección de altmétricas fueron las menciones en medios de noticias y blogs; lecturas en Mendeley, menciones en Wikipedia y Videos considerados por Almetric.com, agrupadas como "Otras fuentes". Todos ellos fueron colectados durante la primera semana de junio, 2020 sin restricción de tipos de contenidos con repercusión mediática, procedencia geográfica o revistas, pero sí por fuentes de origen de los datos.

\subsection{Análisis de datos}

Análisis estadísticos descriptivos (frecuencias) y de correlación (Pearson) fueron empleados para la representación de los datos del estudio. Los datos fueron analizados a partir de los siguientes criterios de selección y variables de evaluación:

- Características de las Sub-categorías ESCI/WoS de Artes y Humanidades en Iberoamérica.

- Coberturas y representatividad por países, tipo de editorial e idioma de publicación.

- Actividad altmétrica: menciones totales en diferentes fuentes sociales, procedencia de repercusión altmétrica por países, revistas con mayor repercusión de artículos publicados y alcance de los artículos por fecha de publicación.

\section{Resultados y discusión}

Los resultados de este estudio muestran que en el conjunto $\mathrm{ESCl}$ existe una representación dispar de ciertos países e idiomas en detrimento de otros en la cobertura de las revistas ubicadas en la Web of Science (Mongeon; Paul-Hus, 2016). La muestra seleccionada sobre aquellas revistas pertenecientes al área geográfica Iberoamericana del área de Arte y Humanidades, representa el $31,86 \%$ del total del área. Que, a su vez, representa el 17,66\% sobre la totalidad de la base de datos ESCl. Por tanto, el área seleccionada tiene una menor representación frente a las Ciencias Sociales y las STEM, en donde un mayor porcentaje de las investigaciones se materializan en la publicación de un artículo.

\subsection{Sub-categorías de Artes y Humanidades}

En la Tabla 1 se presenta el universo de estudio del área Arte y Humanidades de la base de datos $\mathrm{ESCl}$ de los países Iberoamericanos, detallando las 26 sub-categorías del área junto con el 
número de publicaciones de cada país. Los sumatorios reflejan la totalidad de las revistas del área, 442.

En base a la muestra seleccionada, y como era de esperar, no todas las sub-categorías tienen presencia de revistas editadas en Iberoamérica. Varias de ellas poseen una temática muy focalizada que dificulta iniciar un proyecto editorial determinado y mantenerlo en el largo plazo. No es sorprendente que la literatura sobre artes y humanidades plantee problemas únicos. Como dato positivo se dispone de varias sub-categorías en donde las revistas de procedencia Iberoamericana ofrecen una gran visibilidad en el plano internacional, incluso con una cobertura por encima del $70 \%$ a nivel global, es el caso de aquellas centradas en una la temática Literature, Romance. Otras cuatro presentan coberturas superiores al 40\%, son el caso de las especialidades: Classics, Architecture, History y Medieval \& Renaissance Studies. Estos datos demuestran la visibilidad internacional que, en su conjunto, presentan las revistas Iberoamericanas de las áreas de Arte y Humanidades, por las que sigue creciendo el interés para la comunidad académica e investigadora.

Tabla 1. Cobertura de las revistas Iberoamericanas del área de Artes y Humanidades a través de las 26 sub-categorías.

\begin{tabular}{|c|c|c|c|c|c|c|c|c|c|c|c|c|c|c|}
\hline Categoría A\&H & AR & BR & $\mathrm{CL}$ & $\mathrm{co}$ & CR & EC & SV & $\mathrm{MX}$ & PE & UY & VE & PT & ES & Total \\
\hline Architecture & 1 & 1 & 2 & 3 & & 1 & & 1 & & & & & 11 & 20 \\
\hline Art & 1 & & & 1 & & & & 1 & & & & & 20 & 23 \\
\hline \multicolumn{15}{|l|}{ Asian Studies } \\
\hline Classics & 1 & 1 & & & & & & & & & & 2 & 5 & \\
\hline \multicolumn{15}{|l|}{ Dance } \\
\hline Film, Radio, Television & 2 & & & & & & & & & & & 1 & 2 & \\
\hline \multicolumn{15}{|l|}{ Folklore } \\
\hline History & 13 & 17 & 2 & 10 & & 1 & & 5 & & 1 & 3 & 3 & 38 & 93 \\
\hline History \& Philosophy Of Science & & & & 1 & & & & 1 & & & & & 3 & 5 \\
\hline Humanities, Multidisciplinary & 1 & 10 & 4 & 5 & 1 & & 1 & 2 & 1 & & 1 & 5 & 43 & 74 \\
\hline Language \& Linguistics & 2 & 19 & & 1 & & & & 1 & & & 1 & 1 & 29 & 54 \\
\hline \multicolumn{15}{|l|}{ Literary Reviews } \\
\hline Literary Theory \& Criticism & 1 & 3 & & 1 & & & & 1 & & & 1 & & & \\
\hline Literature & 3 & 9 & & 4 & 1 & & & & & & 1 & & 8 & 26 \\
\hline \multicolumn{15}{|l|}{ Literature, African, Australian } \\
\hline \multicolumn{15}{|l|}{ Literature, American } \\
\hline \multicolumn{15}{|l|}{ Literature, British Isles } \\
\hline Literature, German, Dutch, Scandina & & & & & & & & & & & & & 1 & 1 \\
\hline Literature, Romance & 1 & 5 & & 1 & & & & 1 & & & & 1 & 18 & 27 \\
\hline \multicolumn{15}{|l|}{ Literature, Slavic } \\
\hline Medieval \& Renaissance Studies & & & 1 & & & & & & & & & & 16 & 17 \\
\hline Music & & 3 & & & & & & & & & & & 5 & 8 \\
\hline Philosophy & 4 & 9 & 2 & 4 & & & & 3 & & 1 & & 4 & 23 & 50 \\
\hline \multicolumn{15}{|l|}{ Poetry } \\
\hline Religion & 1 & 12 & 1 & & & & & & & & & & 4 & 18 \\
\hline \multirow[t]{2}{*}{ Theater } & & 3 & & 1 & & & & & & & & & 1 & 5 \\
\hline & 31 & 92 & 12 & 32 & 2 & 2 & 1 & 16 & 1 & 2 & 7 & 17 & 227 & 442 \\
\hline
\end{tabular}

Fuente: Elaboración propia.

En relación con la representatividad de la procedencia de las revistas, España concentra la mayor proporción con el 51,4\% de las revistas, seguido de Brasil con el 20,8\%, Colombia y Argentina 
con alrededor del 7\% respectivamente. Los países en el extremo inferior son Costa Rica (2), Ecuador (2), Uruguay (2), El Salvador (1) y Perú (1). Por otro lado, la visibilidad de las revistas publicadas en Iberoamérica frente al resto de países se puede observar en la Figura 1, que muestra la totalidad de las revistas en ambos grupos geográficos. Los valores absolutos destacan varias sub-categorías en donde la presencia Iberoamericana en el plano internacional es muy notable. Dos de las áreas de Artes y Humanidades concentran más del $50 \%$ de la totalidad de las publicaciones: Literature, Romance $(71,79 \%)$ y Classics $(56,25 \%)$. Por encima del $40 \%$ de cobertura despuntan tres subcategorías Architecture (43,75\%); History (46,72\%) y Medieval \& Renaissance Studies (45,94\%). En el lado contrario se aprecia (Tabla 1) 9 sub-categorías en donde el área geográfica estudiada no tiene presencia.

Figura 1: Cobertura IBER y No-IBER de áreas de las revistas Artes y Humanidades en ESCI
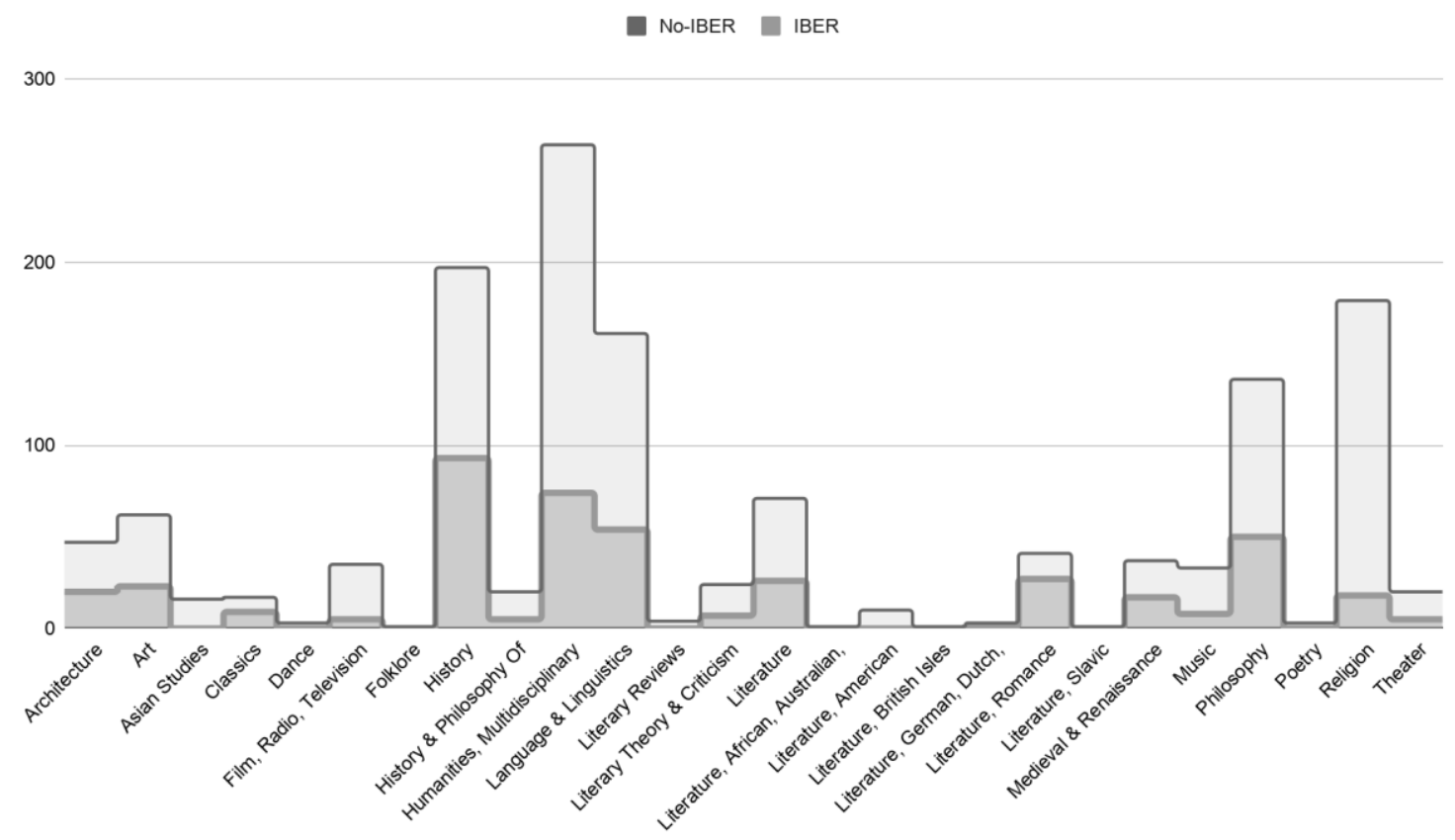

Fuente: Elaboración propia.

\subsection{Instituciones editoras}

El siguiente gráfico muestra las editoriales que más revistas publican del área de Arte y Humanidades pertenecientes a $\mathrm{ESCl}$ en Iberoamérica. Como se puede apreciar, en las primeras posiciones destacan editoriales pertenecen a universidades. Eran de esperar estos resultados ya que en la base de datos Arts \& Humanities Citation Index, el 33,8\% de las revistas se editan por universidades (REPISO; ORDUÑA-MALEA; AGUADED, 2019). 
Destaca la Universidad Complutense de Madrid con 35 títulos, siendo la editorial que más revistas públicas en el área de $A \& H$, seguido de la Universidad de Murcia, con 11 títulos, la Universidad de Coimbra, la Universidad Nacional de La Plata y Autónoma de Barcelona con 8 publicaciones cada una (ver Figura 2).

Ante esta situación se puede afirmar que las universidades poseen una gran fuerza en la disciplina académica de las Artes y Humanidades y esta situación se refleja en el sustancial valor que las universidades ofrecen a las editoriales y revistas de esta área académico científico. Estas grandes editoriales pertenecen, en muchos casos, a grandes e históricas universidades, en donde el desempeño del equipo editorial busca mantener la reputación de las instituciones universitarias. Por otro lado, el alto número de revistas pertenecientes a las universidades refleja que las decisiones editoriales buscan mejorar el impacto y visibilidad de las publicaciones.

Figura 2. Editoriales de revistas iberoamericanas A\&H en ESCl.

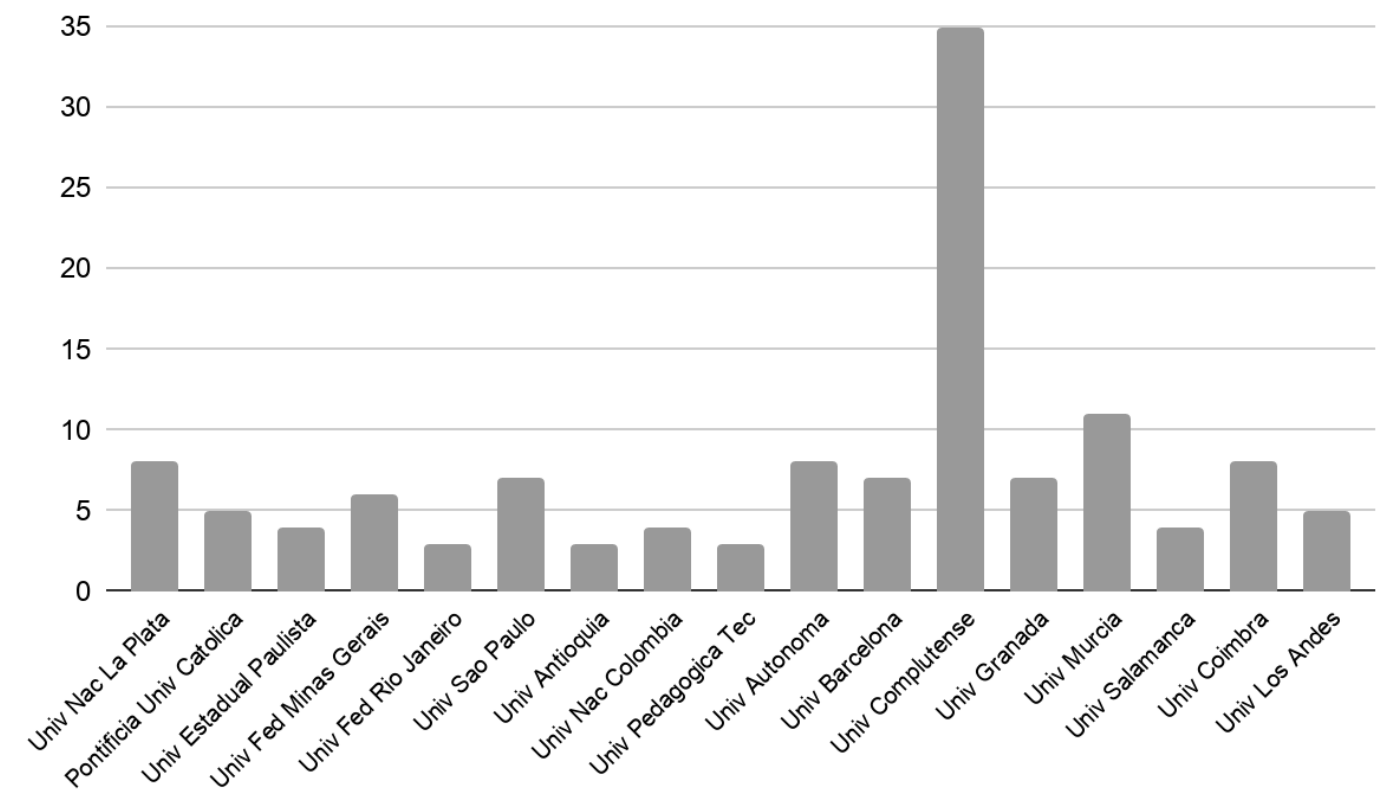

Fuente: Elaboración propia.

Nota: Editoriales con 3 o más revistas.

\subsection{Idiomas de publicación}

En la Tabla 2 se muestran las lenguas más utilizadas por los 7.1852 títulos del conjunto total de $\mathrm{ESCl}$, también presenta una comparación en la cobertura de idiomas por las revistas iberoamericanas frentes a las que no lo son, ambos de A\&H. En ese sentido, de la muestra de revistas de Artes y Humanidades pertenecientes al área geográfica Iberoamericana (442), más de la mitad de las mismas permiten publicar en más de un idioma (233), en cambio, el idioma español 
presenta la segunda opción de títulos frecuentes (153), seguido del portugués (41), inglés (13). En cuanto al resto de revistas (905) pertenecientes al área de Arte y Humanidades, denominadas "No Iber", la lengua inglesa presenta el mayor grupo con más de la mitad de estas (515), seguido de aquellas revistas que publican en más de un idioma.

Como es de esperar, el inglés tiene una posición dominante en el global de $\mathrm{ESCl}$ (7.852) presentando un comportamiento similar a las revistas no Iberoamericanas de Arte y Humanidades. El inglés está sobrerrepresentado en todas las áreas científicas ya que dos terceras partes (5.252) publican en inglés, seguido del multi-idioma (1.438).

Más del 90\% de las revistas situadas en la WoS publican en inglés o en multi idioma (en donde suele incluirse el inglés dentro de este grupo). Ante esta situación, y como he podido comprobar, alrededor del $55 \%$ de las revistas de Arte y Humanidades Iberoamericanas se encontrarán en situación de cumplir este requisito importante para situarse en bases de datos "superiores". Teniendo en cuenta que el $99 \%$ de la población de lberoamérica no pertenece a países de habla inglesa es un compromiso, por parte de las revistas, adaptarse al idioma aceptado como vehículo científico, la lengua inglesa, incluso cuando suelen centrarse en documentos que abordan temas relevantes a nivel local o regional escritos en idiomas vernáculos.

Tabla 2. Distribución comparativa de idiomas en revistas ESCI de A\&H

\begin{tabular}{|l|r|r|r|r|r|r|r|}
\hline \multicolumn{1}{|c|}{ Idioma } & Iber A\&H & \multicolumn{1}{c|}{$\%$} & No Iber A\&H & $\%$ & Global ESCI & $\%$ \\
\hline Multi-idioma & 233 & 52,71 & 313 & 33,12 & 1.438 & 18,3 \\
\hline Español & 153 & 34,62 & 2 & 0,21 & 613 & 7,8 \\
\hline Portugués & 41 & 9,28 & 0 & 0,00 & 148 & 1,9 \\
\hline Inglés & 13 & 2,94 & 515 & 54,50 & 5.252 & 66,9 \\
\hline Otros & 2 & 0,45 & 115 & 12,17 & 401 & 5,1 & 100 \\
\hline Total & 442 & 100 & 945 & 100 & 7.852 & 100 \\
\hline
\end{tabular}

Elaboración propia.

\subsection{Repercusión altmétrica a nivel de revistas}

Un primer examen desprende que, de las 26 sub-categorías de $A \& H$ analizadas a nivel social, 16 presentan revistas con impacto mediático arrojando ratios porcentuales predominantes para las fuentes de social media (Facebook, Twitter) en la mayoría de las áreas, con el particular comportamiento en revistas de dos áreas donde se muestra cierto impacto también en los medios de noticias y blogs (Classics, 34,8\%; Religion, 17,9\%) y de otras fuentes, en Wikipedia y Videos (Religion, 10,7\%) (ver Figura 3). El resto de sub-categorías o no incluyen revistas de países 
Iberoamericanos (9) o no presenta repercusión altmétrica (como es el caso de la revista Literature, German, Dutch, Scandina).

Figura 3. Repercusión altmétrica de las sub-categorías $A \& H$ de ESCI

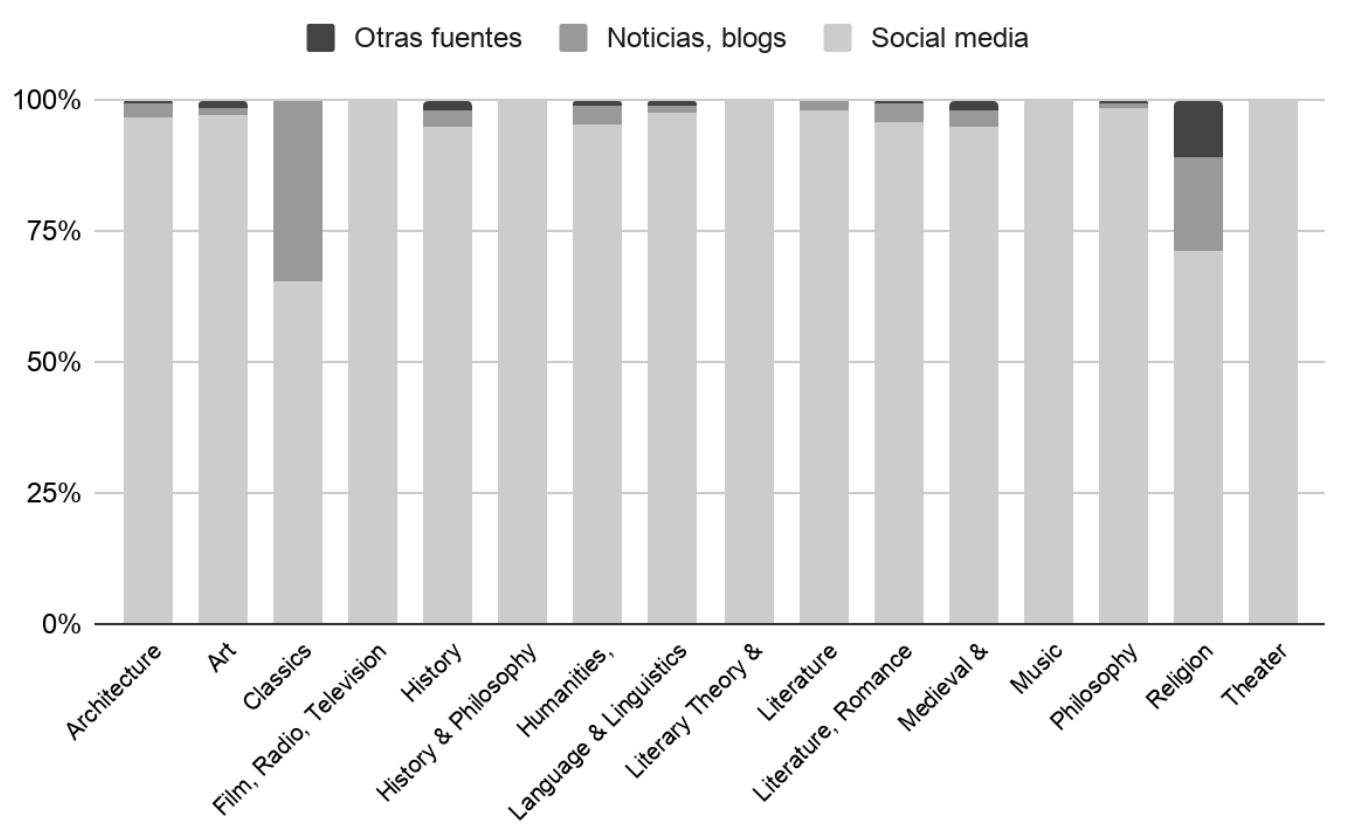

Fuente: Elaboración propia.

En la Figura 4 se identifica la procedencia de la actividad social, donde España aparece como el país más activo seguido de Brasil y Colombia, respectivamente. Además, resulta positivo apreciar que entre los países más dinámicos se encuentren varios externos al área geográfica Iberoamericana: Alemania, Holanda, Gran Bretaña y Estados Unidos. Lo que indica el grado de visibilidad y repercusión de algunas de las revistas del área de Artes y Humanidades.

Figura 4. Cobertura de países que comparten en Facebook, Twitter y Noticias* 


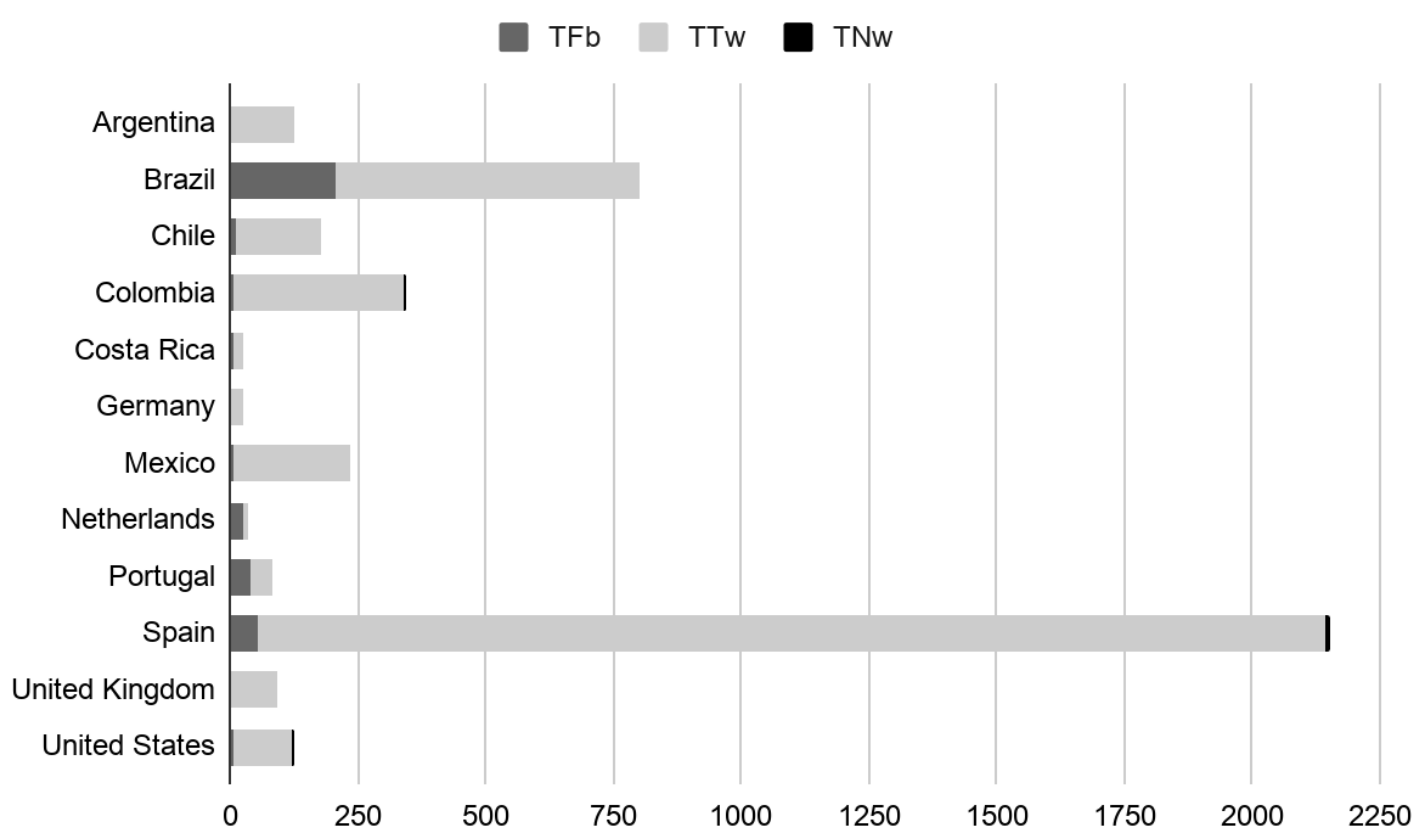

Fuente: Elaboración propia.

*Nota: TFb, total de menciones en Facebook; TTw, total menciones en Twitter; TNw, total de entradas en páginas de noticias.

En la Figura 5 se presentan los datos de impacto mediático en dos plataformas de redes sociales (Facebook y Twitter) y uno de marcado de lectura o bookmarking (Mendeley), obtenidos de 2.751 artículos con alguna atención altmétrica. En un estudio a partir de 12 revistas de Ciencias de la Información de Brasil incluidas en BRAPCI se reporta una muy baja repercusión en plataformas sociales (facebook, twitter), Mendeley y blogs: apenas 0,3\% de los artículos publicados tienen menciones (MANHIQUE et al., 2019).

Así también, una breve aproximación a partir de la fecha cuando se publicó el artículo con al menos una repercusión mediática muestra que los artículos más recientes desde el 2010 a la fecha favorecen a la presencia en Mendeley y en el último lustro para Twitter, dejando el legado, pero constante la repercusión en Facebook.

Figura 5. Repercusión de los artículos por fecha de publicación 


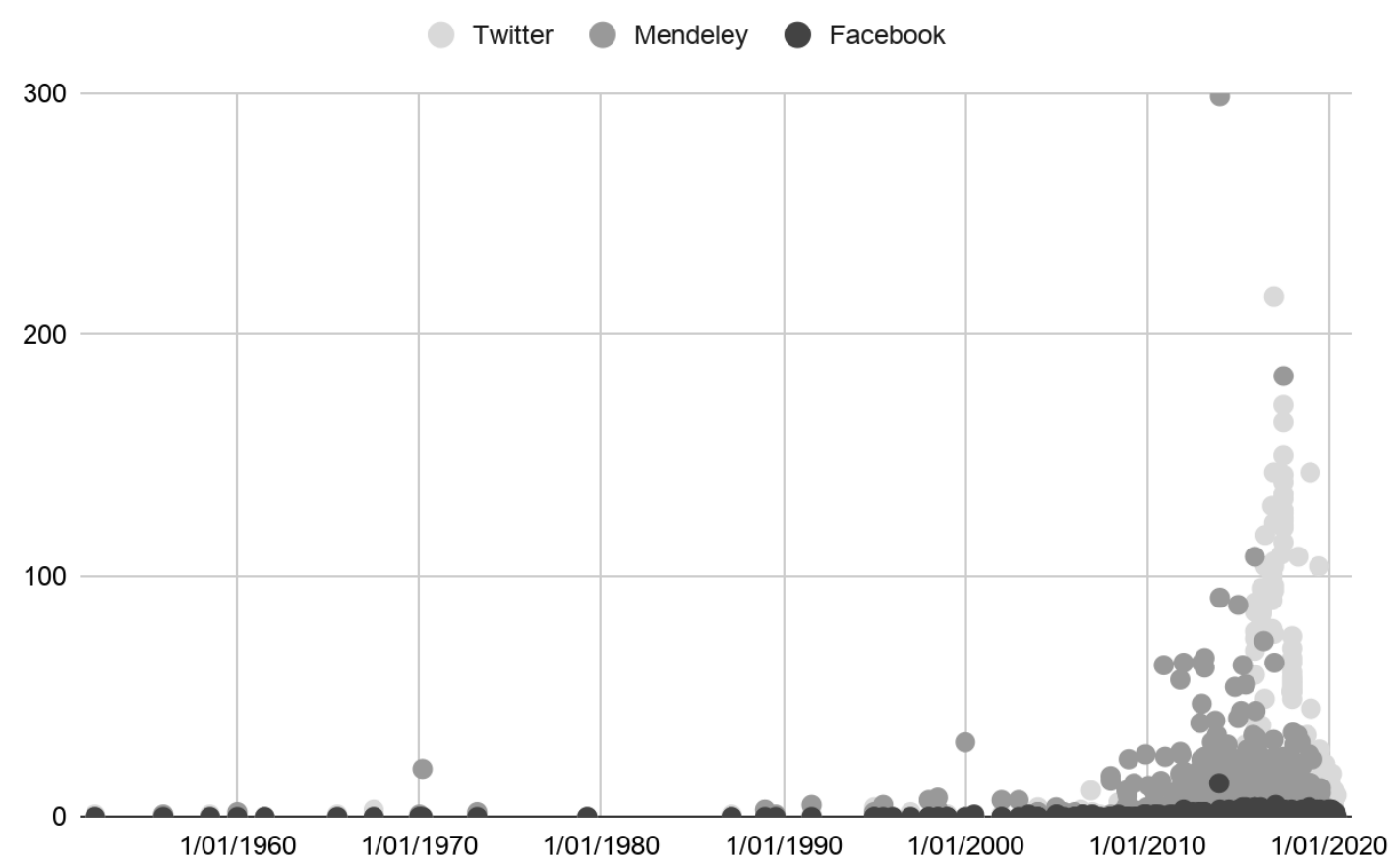

Fuente: Elaboración propia.

Por otra parte, si nos adentramos a observar el impacto mediático de las revistas de Artes y Humanidades provenientes de ESCl, los datos muestran que solo 194 revistas (45.9\%) poseen alguna repercusión o menciones en medios sociales. Resultados similares se encontraron en revistas sudafricanas de acceso abierto donde el $44.6 \%$ de revistas estudiadas por ONYANCHA (2017) obtuvieron alguna mención en medios altmétricos. Asimismo, al desagregar los resultados según fuentes, se encontró que las menciones en Facebook (media=6,52; máx.: 132) y Twitter (media=65,18 máx.: 7,041) son el medio donde las revistas tienen mayor repercusión mediática en redes sociales. En el otro extremo y con escasa presencia de contenidos en medios aparecen los soportes News (media=0,19: máx.: 10) y Blogs (media=0,65; máx.: 29).

En la Tabla 3 se aprecian las veinte revistas, con más artículos, con presencia en medios sociales en donde diez revistas proceden de España, cuatro de Brasil y el resto pertenecen a otros países de Iberoamérica.

Tabla 3. Revistas con mayor repercusión de artículos en medios altmétricos* 


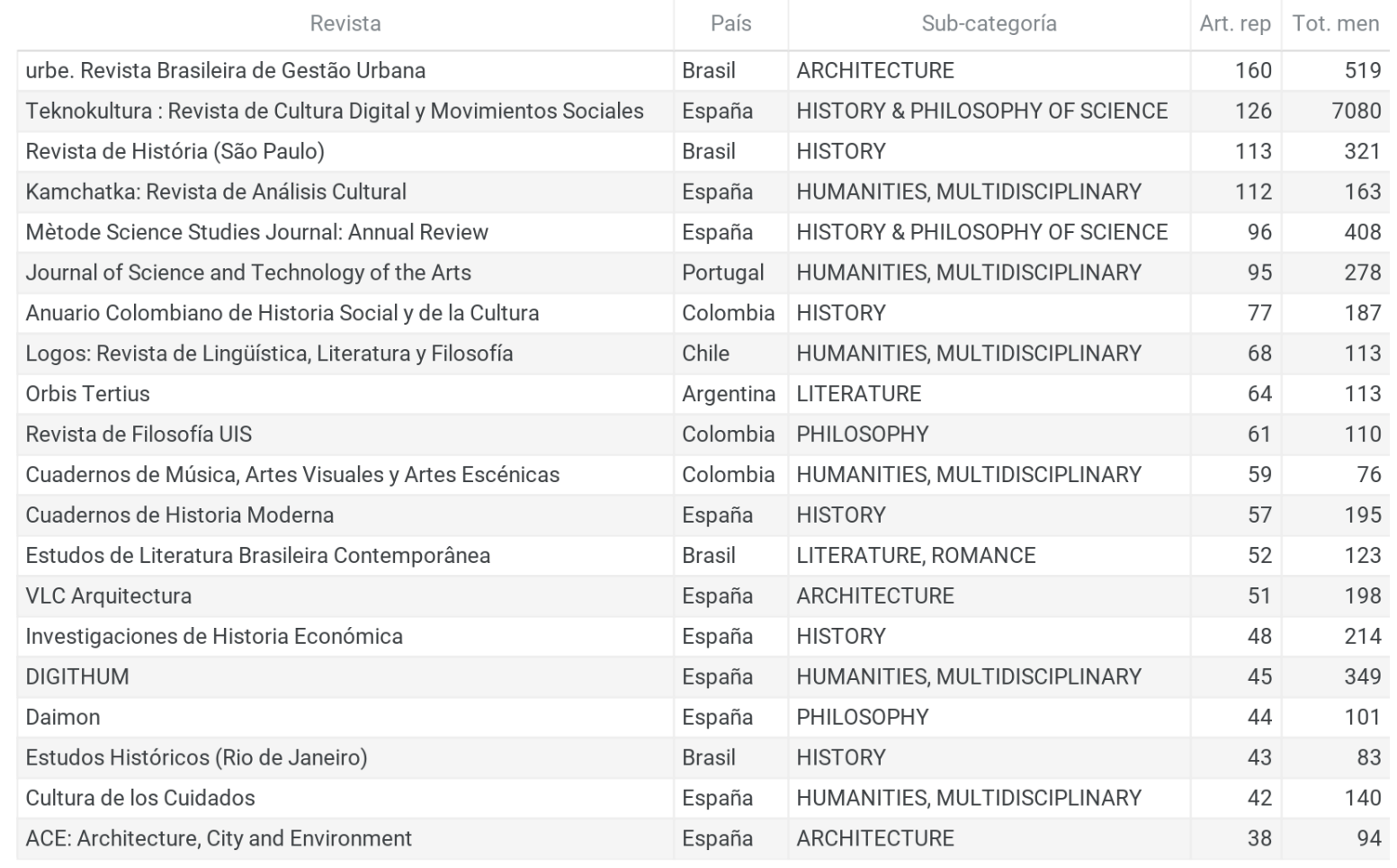

Fuente: Elaboración propia.

*Nota: Art. rep: artículos con repercusión; Tot. rep: total de menciones.

Finalmente, los datos de correlación de las menciones totales en fuentes de Noticias, Blogs, Twitter, Facebook y Wikipedia para las revistas iberoamericanas de A\&H evidencian una moderada correlación positiva entre la repercusión acumulada de las revistas en Twitter y Wikipedia ( $r=0.54)$, además, se reporta baja asociación en los datos de impacto medido por la difusión de los artículos en sitios de noticias frente a las entradas en blogs $(r=0.34)$ y publicaciones en páginas públicas de Facebook ( $r=0.42)$, respectivamente (ver Tabla 4). Los resultados encontrados de la presencia en blogs y similitud con la presencia en prensa digital (news) se asemejan a los datos reportados en revistas de Comunicación del Journal Citation Reports, Web of Science (REPISO; CASTILLOESPARCIA; TORRES-SALINAS, 2019).

Tabla 4. Correlación por fuentes de repercusión altmétrica en revistas de A\&H iberoamericanas 


\begin{tabular}{|l|r|r|r|r|r|}
\hline & 1 & 2 & 3 & 4 & 5 \\
\hline 1. News & 1,00 & 0,34 & 0,13 & 0,42 & 0,32 \\
\hline 2. Blogs & & 1,00 & 0,04 & 0,15 & 0,20 \\
\hline 3. Twitter & & & 1,00 & 0,12 & 0,54 \\
\hline 4. Facebook & & & & 1,00 & 0,29 \\
\hline 5. Wikipedia & & & & & 1,00 \\
\hline
\end{tabular}

\section{Conclusiones}

Iberoamérica concentra el $31.86 \%$ de las revistas del área de Artes y Humanidades del índice ESCl global. De las 26 sub-categorías de WoS que responden a las Artes Humanidades 17 áreas integran revistas de América Latina, El Caribe, España y Portugal, algunas de ellas con un gran número de títulos, por ejemplo 4 poseen más de 50 publicaciones: History (93 publicaciones), Humanities, Multidisciplinary (74), Language \& Linguistics (54) y Philosophy (50).

Las nueve sub-categorías de $A \& H$ que no tienen revistas de procedencia geográfica Iberoamericana, fundamentalmente es debido a la especificidad de los contenidos temáticos que favorecen los estudios a lugares remotos de Iberoamérica y por tanto resultaría difícil que se inicien proyectos editoriales; por ejemplo: Asian Studies; Dance; Literary Reviews; Literature, African, Australian; Literature, American, Literature, British Isles, Literature, Slavic y Poetry.

El idioma empleado por las revistas Iberoamericanas indica la tendencia internacional del sector estudiado, puesto que más de la mitad de las revistas publica los artículos más de un idioma, pudiendo ser inglés, español y portugués.

La valoración por parte de las universidades de las $A \& H$ queda patente por la alta representación de las editoriales universitarias en la publicación de las revistas Iberoamericanas de esta disciplina académica. Estos entornos facilitan la creación de investigadores de carrera temprana al contar en su propia institución con una variedad de publicaciones periódicas, ayudando y definiendo su futuro hacia carreras artísticas, humanísticas, investigadoras, académicas u otras.

En el campo altmétrico, no todas las sub-categorías de A\&H poseen presencia en medios sociales como era de esperar. Las más activas presentan impacto mediático predominantemente para las fuentes de social media de Facebook y Twitter. La red social Twitter comparte contenido de artículos publicados desde el año 2010 fundamentalmente; en cambio Facebook amplía el rango temporal.

Siendo Brasil es uno de los países que comparte más contenidos de Artes y Humanidades en Facebook, mientras que España y Colombia utilizan más el Twitter como herramienta social para la difusión de artículos publicados por revistas de países iberoamericanos. A su vez estos 3 países presentan las revistas con mayor repercusión de artículos en medios altmétricos. 
La manifestación, en las redes sociales, de países externos al área geográfica Iberoamericana (Alemania, Holanda, Gran Bretaña y Estados Unidos) denota el interés y repercusión del contenido Iberoamericano en un plano más amplio

En definitiva, la difusión de una gran parte del conocimiento de las revistas Iberoamericanas en el área de Artes y Humanidades presentes en el índice ESCl está garantizada. Los resultados de los análisis realizados estiman una más que aceptable visibilidad, tanto por el alcance de la divulgación como por la recepción por parte de la comunidad científica.

\section{Agradecimientos}

Agradecemos a Clarivate Analytics por los datos proporcionados de las revistas Emerging Source Citation Index-ESCl y a Altmetric.com por los accesos proporcionados al recurso Altmetric Explorer para investigadores.

\section{Referencias}

ARCHAMBAULT, É.; VIGNOLA-GAGNÉ, É.; CÔTÉ, G.; LARIVIÈRE, V.; \& GINGRAS, Y. Benchmarking scientific output in the social sciences and humanities: The limits of existing databases. Scientometrics, v. 68, n. 3, p. 329-342, 2006.

BESHYAH, S. A. Authors' Selection of Target Journals and Their Attitudes to Emerging Journals: A survey from two developing regions. Sultan Qaboos University Medical Journal, v. 19, n.1, e51, 2019.

BRODERICK, N. A.; CASADEVALL, A. Meta-Research: Gender inequalities among authors who contributed equally. Elife, v. 8, 2019.

BUCHWALD, N. Thinking of information through the humanist's eyes. 1999. Disponível em: http://www.library.arizona.edu/users/buchwaln/humanities.html

COMUNICAR. Ranking de Revistas ESCI de Comunicación y de Educación, 2018. Disponível em: https://www.revistacomunicar.com/index.php?ranking-revistas-esci-country-view=ES

FINKENSTAEDT, T. Measuring research performance in the humanities. Scientometrics, v. 19, n. 56, p. 409-417, 1980.

GARFIELD, E. Is information retrieval in the arts and humanities inherently different from that in science? The effect that ISI's citation index for the arts and humanities is expected to have on future scholarship. Library Quarterly, v. 50, p. 40-57, 1980.

HAMMARFELT, B. Beyond coverage: Toward a bibliometrics for the humanities. In: Research Assessment in the Humanities. Springer: Cham, 2016. p. 115-131.

HEILAND, D.; HUBER, M. T.; KANTER, M. J. Calls to action for the Arts and Humanities in the US. Arts and Humanities in Higher Education, v. 13, n. 1-2, p. 9-16, 2014. 
JABBEHDARI, S.; WALSH, J. P. Authorship norms and project structures in science. Science, Technology, \& Human Values, v. 42, n. 5, p. 872-900, 2017.

LEYDESDORFF, L.; HAMMARFELT, B.; SALAH, A. The structure of the Arts \& Humanities Citation Index: A mapping on the basis of aggregated citations among 1,157 journals. Journal of the American Society for Information Science and Technology, v. 62, n.12, p. 2414-2426, 2011.

LEYDESDORFF, L.; SALAH, A. A. A. Maps on the basis of the Arts \& Humanities Citation Index: The journals Leonardo and Art Journal versus "digital humanities" as a topic. Journal of the American Society for information Science and Technology, v. 61, n.4, p. 787-801, 2010.

MANHIQUE, I. L. E.; RODRIGUES, F. DE A.; SANT'ANA, R. C. G.; CASARIN, H. DE C. S. Indicadores altmétricos em periódicos brasileiros da Ciência da Informação. Revista Ibero-Americana de Ciência da Informação, v. 12, n.2, p. 515-532, 2019.

MIQUEL-SEGARRA, S. Análisis de la investigación publicada en las revistas de comunicación con índice ESCl (Emerging Source Citation Index). En: Tendencias metodológicas en la investigación académica sobre comunicación. España: Comunicación Social, 2018, p. 109-130.

МОСКАЛЕВА, О. В., \& ПИСЛЯКОВ, В. В. Российские журналы в Emerging Sources Citation Index. In: INTERNATIONAL SCIENTIFIC AND PRACTICAL CONFERENCE "WORLD-CLASS SCIENTIFIC PUBLICATION: BEST PRACTICES IN PREPARATION AND PROMOTION OF PUBLICATIONS", 6, 2017, Moscow. Proceedings International Scientific and Practical Conference. 2017. p. 78-81. Disponível em: http://dx.doi.org/10.24069/2017.978-5-7996-2227-5.13

MONGEON, P.; PAUL-HUS, A. The journal coverage of Web of Science and Scopus: a comparative analysis. Scientometrics, v. 106, n.1, p. 213-228, 2016.

NEDERHOF, A. J. Bibliometric monitoring of research performance in the social sciences and the humanities: A review. Scientometrics, v. 66, n. 1, p. 81-100, 2006.

NWAGWU, W.; EGBON, O. Bibliometric analysis of Nigeria's social science and arts and humanities publications in Thomson Scientific databases. The Electronic Library, v. 29, n. 4, p. 438-456, 2011.

ONYANCHA, O. B. Altmetrics of South African journals: implications for scholarly impact of South African research. Publishing Research Quarterly, v. 33, n. 1, p. 71-91, 2017.

PRIEM, J.; PIWOWAR, H.; HEMMINGER, B. H. Altmetrics in the wild: Using social media to explore scholarly impact. ArXiv: 1203.4745v1, 2012.

PRIEM, J.; TARABORELLI, D.; GROTH, P; NEYLON, C. Altmetrics: a manifesto. Disponível em: http://altmetrics.org/manifesto/, 2010.

REPISO, R. Impacto de las revistas emergentes de Bibliotecología y Ciencia de la Información en Web of Science. Bibliotecas. Anales de investigación, v. 15, n. 1, p. 126-129, 2019.

REPISO, R.; JIMÉNEZ-CONTRERAS, E.; AGUADED, I. Revistas Iberoamericanas de Educación en SciELO Citation Index y Emerging Source Citation Index. Revista Española de Documentación Científica, v. 40, n. 4, p. 1-13, 2017.

REPISO, R.; CASTILLO-ESPARCIA, A.; TORRES-SALINAS, D. Altmetrics, alternative indicators for Web of Science Communication studies journals. Scientometrics, v. 119, n. 2, p. 941-958, 2019. 
REPISO, R.; ORDUÑA-MALEA, E.; AGUADED-GÓMEZ, J. I. Revistas científicas editadas por universidades en Web of Science: características y contribución a la marca universidad. EI profesional de la información, v. 28, n. 4, 2019.

RUIZ PÉREZ, R.; JIMÉNEZ-CONTRERAS, E. Los Emerging Sources Citation Index y la internacionalización de las revistas científicas españolas, con especial mención a las de Psicología. Psicothema, v. 31, n. 4, p. 376-383, 2019.

SEGADO-BOJ, F.; MARTÍN-QUEVEDO, J.; PRIETO-GUTIÉRREZ, J. J. Attitudes toward open access, open peer review, and altmetrics among contributors to Spanish scholarly journals. Journal of scholarly publishing, v. 50, n. 1, p. 48-70, 2018.

SUGIMOTO, C. R.; WORK, S.; LARIVIÈRE, V.; HAUSTEIN, S. Scholarly use of social media and altmetrics: A review of the literature. Journal of the Association for Information Science and Technology, v. 68, n. 9, p. 2037-2062, 2017.

THELWALL, M. Altmetric Prevalence in the Social Sciences, Arts and Humanities: Where are the Online Discussions?. Journal of Altmetrics, v. 1, n. 1, 2018.

VÍLCHEZ-ROMÁN, C.; HUAMÁN-DELGADO, F; ALHUAY-QUISPE, J. Social dimension activates the usage and academic impact of Open Access publications in Andean countries: a structural modelingbased approach. Information Development, 2020. Disponível em: https://doi.org/10.1177/0266666920901849

WANG, W. M.; HO, Y. S. Bibliometric analysis of art exhibit reviews in the Arts \& Humanities Citation Index. Malaysian Journal of Library \& Information Science, v. 22, n.1, p. 59-68, 2017.

WOUTERS, P.; COSTAS, R. Users, narcissism and control: Tracking the impact of scholarly publications in the 21st century. Utrecht: SURF foundation. 2012. Disponível em: http://www.surffoundation.nl/nl/publicaties/Documents/Users\%20narcissism\%20and\%20control. pdf 